\title{
DIVERGÊNCIA NO CRESCIMENTO FOLICULAR: EFEITO NA COMPETÊNCIA OOCITÁRIA PARA PRODUÇÃO IN VITRO DE EMBRIÕES - REVISÃO
}

\author{
(Follicular growth divergence: effect in the oocyte competence for \\ in vitro embryo production)
}

\author{
CASTILHO, C. ${ }^{1,2}$; GARCIA, J.M. ${ }^{1}$ \\ 'Depto. de Medicina Veterinária Preventiva e Reprodução Animal, FCAV/UNESP, Jaboticabal-SP; \\ 2Docente Faculdade de Ciências Agrárias - Curso de Medicina Veterinária e Zootecnia UNOESTE - \\ Presidente Prudente-SP.
}

\begin{abstract}
RESUMO - O crescimento do oócito dentro do folículo ovariano é determinado por vários fatores que influenciam sua viabilidade e competência para o desenvolvimento in vitro. Estes fatores incluem tamanho folicular, dia do ciclo estral, nível de atresia e influência de outros folículos, tal como o folículo dominante (FD). A fase de seleção do FD é visualizada por ultrasonografia como um rápido aumento no diâmetro do FD, enquanto os outros folículos da mesma onda cessam seu crescimento e/ou regridem. Estas mudanças que ocorrem no diâmetro refletem as importantes alterações endócrinas, parácrinas e autócrinas que acompanham ou antecedem este momento crítico do desenvolvimento folicular e, por sua vez, podem influenciar a qualidade do oócito e a competência para o desenvolvimento in vitro. A aspiração folicular conduzida por ultra-sonografia e posterior fecundação in vitro dos oócitos obtidos de doadoras vivas e de alto valor genético tem sido uma técnica em franca ascensão no mundo, sobretudo devido ao importante incremento no melhoramento genético do rebanho. Porém, existe variabilidade nos resultados do desenvolvimento in vitro de embriões bovino e, em média, apenas $30 \%$ a $40 \%$ dos oócitos maturados se desenvolvem até o estádio de blastocisto.
\end{abstract}

Palavras-chave: divergência folicular, folículo dominante, aspiração folicular, oócito.

ABSTRACT - The oocyte growth in the ovarian follicle is determined by several factors which influence its viability and ability for the in vitro development. These factors include: follicular size, estrous cycle day, atresy level and influence of other follicles, such as dominant follicle (DF). The phase of DF selection is visualized by ultrasound as it increases in the diameter of the DF, while other wave follicles stop their growth or undergo regression. These changes that occur in the diameter reflect the important endocrine, paracrines and autocrines alterations that follow or precede this critical moment of the folicle development; in turn, they can influence the oocyte quality and the ability to the in vitro development. The ovum pick-up following in vitro fertilization (IVF) of the oocyte from gotten alive givers and high genetic value has been one of the expanding technique in the world, over all regard to the important genetic increment in the improvement of the flock. However, variability in the results of the in vitro embryo bovine development exists and, in average, only $30 \%$ to $40 \%$ of the matured oocyte will develop until blastocyst.

Key-words: follicular deviation, dominant follicle, OPUIVF, oocyte.

\section{Introdução}

A obtenção de embriões pela técnica de produção in vitro tem crescido nos últimos anos, sobretudo em bovinos onde está mais evoluída. O incremento na proporção de embriões produzido in vitro, oriundo de oócitos colhidos de doadoras vivas pela aspiração folicular guiada por ultra-sonografia (Ovum Pick-up, OPU), tem sido utilizado, no entanto a proporção de embriões produzidos ainda permanece baixo. Portanto, para otimizar a utilização da aspiração folicular em termos de número e qualidade dos complexos cumulus oócitos (COCs) recuperados e a competência para o desenvolvimento in vitro é necessário conhecer melhor a fisiologia reprodutiva da fêmea bovina, principalmente no que diz respeito à foliculogênese e seu controle endócrino.

\section{Dinâmica Folicular}

O processo contínuo de crescimento e atresia dos folículos que leva ao desenvolvimento do folículo préovulatório é conhecido como dinâmica folicular, enquanto que o padrão de crescimento e atresia de um grupo de folículos ovarianos é denominado onda de crescimento folicular (LUCY et al., 1992). A dinâmica folicular em novilhas ou vacas zebuínas e européias tem sido caracterizada desde a década de 80 pela ultrasonografia diária e apresenta-se em um padrão de duas (PIERSON e GINTHER, 1988; GINTHER et al., 1989; FIGUEIREDO et al., 1997) ou três (SAVIO et al., 1988; 
GAMBINI et al., 1998; CASTILHO et al., 2000) ondas de crescimento folicular durante o ciclo estral.

Cada onda folicular é composta por uma fase de recrutamento, no qual um grupo de folículos primordiais e primários inicia seu crescimento. Dentre estes, um folículo é selecionado, não sofre atresia e potencialmente pode chegar a ovular (fase de seleção). O folículo selecionado passa a exercer dominância (fase de dominância) sobre os demais folículos, suprimindo o crescimento dos mesmos e inibindo o recrutamento de um novo grupo de folículos (GINTHER et al., 1996; GINTHER et al.,1989; SIROIS e FORTUNE, 1990).

A seleção do FD capaz de ovular dentro de um grupo de folículos antrais é um processo dinâmico regulado por interações entre gonadotrofinas, fatores de crescimento e substâncias intra-ovarianas (AUSTIN et al., 2001; YUAN et al., 1998), resultando em uma fase de transição crítica para o desenvolvimento folicular.

\section{Divergência no crescimento folicular}

O sinal mais evidente para a seleção folicular é observado pela ultra-sonografia ovariana seriada como uma divergência no diâmetro do folículo dominante (FD) em relação ao maior folículo subordinado (FS), ou seja o FD continua crescendo, ao passo que os FS diminui ou cessa seu crescimento. O termo divergência foi primeiramente proposto e definido por GINTHER et al. (1996) como o momento no qual se observa o início da maior diferença nas taxas de crescimento entre o futuro FD e o maior subordinado.

Em novilhas da raça Holandesa, após mensurações seriadas do diâmetro dos dois maiores folículos ovarianos, foi observado que a divergência na taxa de crescimento ocorre em média 2,8 dias após a emergência da primeira onda folicular, quando o futuro FD apresenta um diâmetro médio de $8,5 \mathrm{~mm}$ e o maior FS 7,2 mm (GINTHER et al., 1996 e 1997). O futuro FD de $3 \mathrm{~mm}$ foi detectado em média 6 horas antes do maior subordinado, sendo que os dois demonstraram um crescimento paralelo até a divergência (GINTHER at al., 1997 e 1999).

Estes resultados sugerem que o mecanismo de divergência foi ativado quando o futuro FD alcançou um diâmetro determinante $(8,5 \mathrm{~mm})$, o qual foi concluído antes que o FS alcançasse um diâmetro similar (KULICK et al., 1999). Em novilhas da raça Nelore, CASTILHO (2003) estudando a seleção folicular observou que em média 84 horas após a ovulação o maior folículo subordinado medindo $5,5 \mathrm{~mm}$ cessa seu crescimento, enquanto o futuro dominante com $7,5 \mathrm{~mm}$ continua a se desenvolver.

\section{Hormônio Folículo Estimulante (FSH)}

Existe uma relação entre a concentração de $\mathrm{FSH} e$ a seleção do FD, quando a concentração de $F S H$ declina o FD continua se desenvolvendo. Existe um aumento na concentração sérica de FSH antecedendo em 1 a 2 dias a emergência de cada onda, o qual diminui após a seleção do FD (ADAMS et al.,1992; GIBBONS et al., 1999). Em novilhas da raça Nelore, 24 horas antes da divergência no crescimento folicular, durante a primeira onda folicular, observou-se a menor concentração plasmática de FSH (CASTILHO, 2003). As concentrações de FSH são mantidas em níveis basais até o FD perder sua dominância, resultando em novo aumento nos níveis de FSH e subseqüente emergência da próxima onda folicular (BODENSTEINER et al., 1996).

Alguns trabalhos têm demonstrado que qualquer folículo saudável ou em crescimento é capaz de se tornar dominante. Tratamentos com FSH no início do desenvolvimento folicular estimulam muitos folículos a obterem um diâmetro de dominância (ADAMS et al., 1993), sendo a base dos protocolos de superovulação com FSH para colheita de embriões. Além disso, o aumento da concentração sérica de FSH, decorrente da destruição do FD após a divergência, resulta na dominância do maior FS (KO et al., 1991, GINTHER et al., 2000a). Da mesma forma, um folículo aleatoriamente escolhido dentre um grupo de folículos de $5 \mathrm{~mm}$, no início da onda folicular, pode ser direcionado para a dominância após a aspiração de todos os outros da onda (GIBBONS et al.,1997). O potencial para dominância no esperado início da divergência é maior nos folículos maiores e decresce progressivamente com o diâmetro, estando ausente nos folículos menores de $7 \mathrm{~mm}$ (GINTHERr et al., 2001).

Talvez todos os folículos recrutados na mesma onda possuam habilidade intrínseca para tornar-se dominante, porém ao se aproximar da divergência, esta capacidade se mantém apenas naqueles que alcançam primeiro diâmetros maiores.

\section{Supressores de FSH}

A produção de estradiol e inibina, sobretudo pelas células da granulosa do FD, atua inibindo a liberação de FSH pela hipófise anterior (FINDLAY e CLARKE, 1987). Parece que estes hormônios são os principais responsáveis pela supressão das concentrações sistêmicas de FSH para níveis abaixo do limiar necessário para manter o crescimento dos folículos subordinados, os quais entram em atresia (DRIACOURT, 1991).

\section{Inibina}

Além do seu efeito na supressão do FSH, o papel preciso da inibina na seleção do FD não está completamente elucidado. No entanto, formas de ativina e inibina sintetizadas pelas células da granulosa estão envolvidas na produção de andrógenos pelas células da teca (FINDLAY, 1993). Em novilhas, MIHM et al., (2000), não observaram qualquer diferença nas concentrações de folistatina, inibina-A e várias formas de inibina com diferentes pesos moleculares no fluido 
folicular dos três maiores folículos quando o maior deles atingiu 7,6 mm. Em outro estudo, usando o momento do esperado início da divergência como referência $(8,5$ $\mathrm{mm}$ ), também não houve diferença na quantidade total de inibina e inibina-A entre o FD e o maior subordinado (BEG et al., 2001). Por outro lado, AUSTIN et al. (2001) observaram aumento significativo de formas precursoras da inibina $(>48 \mathrm{kDa})$ no maior folículo, porém apenas no início da onda (5 a 33 horas após pico de FSH), mantendo-se igual nos 4 maiores folículos após a divergência e queda na concentração de FSH.

\section{Estradiol}

Em novilhas, observou-se aumento na concentração sérica de estradiol no início da divergência. $O$ aumento ou decréscimo na concentração de estradiol neste ponto resulta em decréscimo ou aumento, respectivamente, na concentração de $\mathrm{FSH}$, indicando uma relação funcional entre estes dois hormônios (KULICK et al., 1999; GINTHER et al., 2000b).

Além de atuar na regulação da liberação de FSH, algumas ações locais do estradiol no ovário incluem: estímulo do crescimento folicular (GOLDENBERG et al., 1972); aumento da sensibilidade da granulosa às gonadotrofinas, aumento na expressão de receptores de LH e atividade da enzima P-450 aromatase (HILLIER, 1991); proliferação celular (BLEY et al., 1997) e proteção destas células contra apoptose (MURDOCH, 1998).

Vários autores têm detectado aumento de estrógeno no fluído folicular do folículo destinado a ser o dominante. Estudos utilizando folículos de diâmetros em torno da divergência folicular (8 a $10 \mathrm{~mm}$ ) demonstram maiores concentrações de estradiol no fluido folicular do FD quando comparado ao subordinado (SUNDERLAND et al., 1994; EVANS et al., 1997; KULICK et al., 1999). Recentemente, MINHN et al, (2000) ao dosar estrógeno dos três maiores folículos no dia 3 do ciclo, ou seja 1,5 dias após a emergência da onda, observaram aumento na concentração de estradiol no maior deles $(7,6 \mathrm{~mm})$ quando comparado ao primeiro $(6,9 \mathrm{~mm})$ e segundo $(6,1 \mathrm{~mm})$ folículos subordinados.

Existem evidências de que o estradiol atua como um facilitador local para a mudança na responsividade do maior folículo do FSH para o LH (GONG et al., 1996) no momento que ocorre queda na concentração sérica de FSH.

\section{Receptores de LH na Célula da Granulosa}

Acredita-se que a aquisição de receptores para LH $(\mathrm{LHr})$ tem um importante papel na manutenção do crescimento do folículo selecionado após a queda nos níveis de FSH.

Alguns estudos sobre aquisição de LHr nas células da granulosa iniciaram-se com XU et al., (1995), quando demonstraram que RNAm para LHr começam a ser expressos nas células da granulosa no dia 4 do ciclo estral (dia 0 = dia da emergência), em folículos com 10,8 mm de diâmetro estando ausente em folículos de $6,8 \mathrm{~mm}$ no dia 2 do ciclo. Posteriormente, BAO et al., (1997) comprovaram o achado anterior e observaram que em folículos maiores com 13,0 e 15,0 mm a expressão de RNAm para $\mathrm{LHr}$ (detectáveis pela hibridização in situ) nas células da granulosa destes folículos foi 2 e 4 vezes maior, respectivamente, quando comparada a folículos de $10,8 \mathrm{~mm}$ de diâmetro. Por outro lado, EVANS et al., (1997) demonstraram aumento na concentração de estradiol no FD em relação ao subordinado nos dias $2(8,0 \mathrm{~mm})$ e $3(9,7 \mathrm{~mm})$ do ciclo estral, entretanto sem níveis detectáveis de RNAm (hibridização in situ) para LHr nas células da granulosa destes folículos. Recentemente, BEG et al., (2001) observaram uma diferença na expressão de RNAm de LHr nas células da granulosa pela RT-PCR (reação em cadeia da polimerase) entre os dois maiores folículos, em média 8 horas antes de detectar qualquer aumento no diâmetro de divergência ou na concentração de estradiol no fluido folicular.

Uma outra evidência do papel do LH na divergência no diâmetro é observado em vacas após tratamento contínuo com um análogo do $\mathrm{GnRH}$, o qual suprime a secreção pulsátil de LH e o maior folículo não cresce acima de 7 a 9 mm (GONG et al., 1995, FIKE et al., 1997). Recentemente, um estudo avaliou o momento no qual o FD adquire capacidade para ovular após administração de doses crescentes de LH (SARTORI et al., 2001). Neste estudo observou-se que folículos com 7,0 a $8,5 \mathrm{~mm}$ de diâmetro eram incapazes de ovular, mesmo perante altas doses de LH (40 mg) e apenas folículos com no mínimo $10 \mathrm{~mm}$ de diâmetro ovulavam com esta dosagem.

É provável que a seleção do FD seja um processo progressivo e que os estágios iniciais da seleção ocorram antes de uma perceptível diferença no diâmetro (FORTUNE et al, 2001). O mecanismo completo que leva à seleção ou divergência no diâmetro não está completamente definido, mas parece estar claramente associado à aquisição de LHr pelas células da granulosa, ao aumento da concentração circulante de estradiol-17b e ao decréscimo na concentração de FSH.

\section{Aspiração Folicular (OPU)}

Devido à dinâmica folicular em novilhas ou vacas zebuínas e européias ser caracterizada pela presença de duas ou três ondas de desenvolvimento folicular, é possível realizar a recuperação de oócitos por via transvaginal durante todo o ciclo estral, mesmo repetidamente (KRUIP et al., 1994; PIETERSE et al., 1991; BOLS et al., 1995; BUNGARTZ et al., 1995). Os protocolos têm geralmente consistido de uma ou duas aspirações semanais usando animais superovulados ou não (LOONEY et al., 1994; BOLS et al., 1995; BUNGARTZ et al., 1995; HASLER et al., 1995; STUBBINGS e WALTON, 1995). Além disso, a OPU permite a produção de embriões oriundos de vacas com 
problemas reprodutivos adquiridos (LOONEY et al., 1994; HASLER et al., 1995; RODRIGUES e GARCIA, 2000), durante o primeiro trimestre de gestação (BUNGARTZ et al., 1995; MEINTJES et al., 1995) ou em novilhas pré púberes (ARMSTRONG et al., 1994; BROGLIATTI e ADAMS, 1996).

Entretanto, a capacidade de desenvolvimento após a fecundação é limitada e, apesar dos progressos feitos nas condições de cultivo durante a maturação e desenvolvimento embrionário, somente em torno de um terço (LONERGAN et al., 1994, HENDRIKSEN et al., 2000, DAYAN et al., 2000) dos oócitos selecionados morfologicamente antes de serem submetidos a MIV, resultam em embriões viáveis. Também existe uma ampla variação individual na produção de embriões, KRUIP et al., (1994) relataram uma variação entre as doadoras de $9 \%$ a $26 \%$ e HASLER at al., (1995) observaram variação entre $4 \%$ a $33 \%$ e WATANABE (2002) $0,3 \%$ a $13 \%$.

A influência do tamanho folicular na competência oocitária para o desenvolvimento in vitro foi confirmada por LONERGAN et al., (1994), quando verificaram aumento significativo na produção de embriões oriundos de oócitos aspirados de folículos com mais de $6 \mathrm{~mm}$ de diâmetro. PAVLOK et al., (1992), também observaram melhor desempenho na produção de blastocistos quando oócitos de folículos médios (2-4 mm) e grandes $(4-8 \mathrm{~mm})$ foram utilizados em comparação a oócitos de folículos pequenos (1-2 mm). Em revisão, HENDRIKSEN et al., (2000) concluíram que a competência para o desenvolvimento in vitro é maior para oócitos oriundos de folículos maiores que 6-8 $\mathrm{mm}$ quando comparado com folículos de 3-6 mm. No entanto, CAROLAN etal. (1996) não observaram influência do tamanho folicular sobre a maturação do oócito. Resultado similar foi obtido por SENEDA et al., (2001) estudando oócitos oriundos de folículos pequenos ( $<4 \mathrm{~mm}$ ) e grandes $(>4 \mathrm{~mm}$ ) aspirados in vivo.

Com relação ao estádio do desenvolvimento folicular, MACHATKOVÁ et al., (1996) demonstraram que oócitos colhidos nos dias 14 a 16 do ciclo estral apresentavam melhores índices de competência para o desenvolvimento embrionário quando comparados aos aspirados nos dias 7,8 e 9 . Em revisão, HAGEMANN (1999) conclui que o desenvolvimento até blastocisto é significativamente maior em oócitos colhidos durante a fase de crescimento folicular independente do diâmetro do folículo, porém a competência oocitária tendeu a aumentar em oócitos oriundos de folículos maiores. Segundo HENDRIKSEN et al. (2000), o FD afeta negativamente a competência oocitária dos folículos subordinados provavelmente por induzir avanço no estádio de atresia. Menores taxas de recuperação e de blastocisto são observadas quando a OPU é realizada semanalmente em comparação com OPU a cada 3-4 dias (GOODHAND et al., 1999). Possivelmente a alta freqüência nas aspirações previna o estabelecimento do FD (HENDRIKSEN et al., 2000).

CASTILHO (2003), estudando a fase da primeira onda e o diâmetro folicular sobre a competência oocitária em Nelore observaram que não houve efeito do diâmetro folicular sobre a produção de balstocistos, mas houve interação do diâmetro com a fase folicular e concluíram que a competência é melhor nos folículos pequenos no início da onda e melhora nos folículos maiores com o avanço da onda. Em Bos tauru MACHATKOVÁ et al. (2004) comparando oócitos aspirados em fase de crescimento e de dominância folicular observaram taxa de blastocisto significativamente superior em oócitos aspirados na fase de crescimento. Anteriormente, STOCK e SMITH (1996) utilizaram oócitos oriundos de folículos grandes para maturar oócitos aspirados de folículos pequenos e observaram que a taxa de blastocisto foi semelhante às obtidas de oócitos de folículos maiores (6-9 mm). Demonstrando, portanto, que fatores parácrinos secretados pelo oócito e/ou células do cumulus durante a maturação final são importantes para o desenvolvimento pós-clivagem.

Em suma, o oócito para ser fertilizado in vivo é doado pelo folículo saudável, durante uma fase específica do ciclo estral. Ao passo que, os oócitos colhidos para a produção in vitro são obtidos de folículos em diferentes etapas do desenvolvimento e em fases distintas do ciclo estral, portanto, expostos a diferentes concentrações de estradiol, progesterona, LH e FSH. Estes fatores podem afetar a competência oocitária para o desenvolvimento de embriões in vitro (WIT et al., 2000).

No entanto, não há consenso entre os trabalhos para definir um momento adequado da onda para se realizar a aspiração in vivo, pois os resultados variam muito.

\section{Referências}

ADAMS G.P.; MATTERI R.L.; KASTELIC J.P.; KO J.C.H.; GINTHER, O.J. Association between surges of folliclestimulating hormone and the emergence of follicular waves in heifers. Journal of Reproduction and Fertility, Cambridge, v. 94, p.177-88, 1992.

ADAMS G.P.; KOT K.; SMITH C.A.; GINTHER O.J. Selection of a dominant follicle and suppression of follicular growth in heifers. Animal Reproduction Science, Amsterdam, v.30, p.259-271, 1993.

ARMSTRONG D.T.; IRVINE B.J.; EARL C.R.; MCLEAN D.; SEAMARK R.F. Gonadotropins stimulations regimens for follicular aspiration and in vitro embryo productions from calf oocytes. Theriogenology, New York, v.42, p.1227-1236, 1994.

AUSTIN E.J.; MIHM M.; EVANS A.C.O.; KNIGHT P.G.; IRELAND J.L.H.; ROCHE J.F. Alterations in intrafollicular regulatory factors and apoptosis during selection of follicles in the first follicular wave of the bovine estrous cycle. Biology of Reproduction, Madison, v.64, p.839-848, 2001.

BAO B.; GARVERICK H.A.; SMITH G.W.; SMITH M.F.; SALFEN B.E.; YOUNGQUIST R.S. Changes in messenger ribonucleic-acid ecoding $\mathrm{LH}$ receptor, cytochrome $\mathrm{P} 450$ side chain cleavage, and aromatase are associated with recruitment and selection of bovine ovarian follicles. Biology of Reproduction, Madison, v.56, p.1158-1168, 1997. 
Divergência no crescimento folicular: efeito na competência oocitária para produção in vitro de embriões - Revisão

BEG M.A.; BERGFELD D.R.; KOT K.; WILTBANK M.C.; GINTHER O.J. Follicular-fluid factors and granulosa-cell gene expresion associated with follicle deviation in cattle. Biology of Reproduction, Madison, v.64, p.432-441, 2001.

BLEY M.A.; SARAGUETAP.E.; BARAÑAO J.L. Concerted stimulation of rat granulosa cell deoxyribonucleic acid synthesis by sex steroids and follicle-stimulating hormone. Journal of Steroid Biochemistry and Molecular Biology, Oxon, v.62, p.11-19, 1997.

BODENSTEINER K.J.; KOT K.; WILTBANK M.C.; GINTHER O.J. Synchronization of emergence of follicular wave in cattle. Theriogenology, New York, v.45, p.1115-28, 1996.

BOLS P.E.J.; VANDENHEEDE J.M.M.; VAN SOOM A. Transvaginal ovum-pick-up (OPU) in the cow: a new disposable needle guindance system. Theriogenology, New York, v.43, p.677-687, 1995.

BROGLIATTI G.M.; ADAMS G.P. Ultrasound-guided transvaginal oocyte collection in prepurbetal calves. Theriogenology, New York, v.45, p.1163-1176, 1996.

BUNGARTZ L.; LUCAS-HAHN A.; RATH D.; NIEMANN $\mathrm{H}$. Collection of oocytes from cattle via follicular aspiration aided by ultrasound with or without gonadotropin pretreatment and in different reproductive stages. Theriogenology, New York, v.43, p.667-675, 1995.

CAROLAN C.; LONERGAN P.; MONGET P.; MONNIAUX D.; MERMILLOD P. Effect of follicle size and quality on the ability of follicular fluid to support cytoplasmic maturation of bovine oocytes. Molecular Reproduction and Development, New York, v.43, p.477-483, 1996.

CASTILHO C.; GAMBINI A.L.G.; FERNANDES P.; TRINCA L.A.; TEIXEIRA A.B.; BARROS C.M. Synchronization of ovulation in crossbred dairy heifers using gonadotrophinreleasing hormone agonist, prostaglandin $\mathrm{F} 2 \mathrm{a}$ and human chorionic gonadotrophin or estradiol benzoate. Brazilian Journal of Medical and Biological Research, Ribeirão Preto, v.33, n.1, p.91-101, 2000.

CASTILHO C. (2003). Divergência no crescimento folicular em novilhas da raça Nelore e sua influência sobre a competência oocitária para o desenvolvimento embrionário in vitro. Jaboticabal, 87p. TESE (Doutorado) - FCAV - UNESP Jaboticabal.

DAYAN A.; WATANABE M.R.; WATANABE Y.F. Fatores que interferem na produção comercial de embriões $\mathrm{FI}$. Arquivos da Faculdade de Veterinária da UFRGS, Porto Alegre, v.28, p.181-185, 2000.

DRIANCOURT M.A. Follicular dynamics in sheep and cattle. Theriogenology, New York, v.35, p.35-55, 1991.

EVANS A.C.O.; KOMAR C.M.; WANDJI A.S.; FORTUNE J.E. Changes in androgen secretion and luteinizing hormone pulse amplitude are associated with the recruitment and growth of ovarian follicles during the luteal phase of the estrous cycle. Biology of Reproduction, Madison, v.57, p.394-401, 1997.
FIGUEIREDO, R.A.; BARROS, C.M.; PINHEIRO, O.L, SOLER, J.M.P. Ovarian follicular dynamics in Nelore Breed (Bos indicus), Theriogenology, New York, v.47, p.1489-1505, 1997.

FIKE K.E.; BERGFELD E.G.; CUPP A.S.; KOJIMA F.N.; MARISCAL V.; SANCHEZ T.; WEHRMAN M.E.; GROTJAN H.E.; HAMERNIK D.L.; KITTOK R.J.; KINDER J.E. Gonadotropin secretion and development of ovarian follicles during oestrous cycles in heifers treated with luteinizing hormone releasing hormone antagonist. Animal Reproduction Science, Amsterdam, v.49, p.83-100, 1997.

FINDLAY J.K. An update on the roles of inhibin, activin, and follistatin as local regulators of folliculogenesis. Biology of Reproduction, Madison, v.48, p.15-23, 1993.

FINDLAY J.K.; CLARKE I.J. Regulation of the secretion of FSH In domestic ruminants. Journal of Reproduction and Fertility, Cambridge, v.34, p.27-37, 1987.

FORTUNE J.E.; G.M. RIVERA, A.C.O. EVANS, A.M. TURZILLO. Differentiation of dominant versus subordinate follicles in cattle. Biology of Reproduction, Madison, v.65, p.648-654, 2001.

GAMBINI A.L.G; MOREIRA M.B.P.; CASTILHO C.; BARROS C.M. Dinâmica folicular e sincronização da ovulação em vacas da raça Gir. Revista Brasileira de Reprodução Animal, Belo Horizonte, v.22, p.201-210, 1998.

GIBBONS J.R.; WILTBANK M.C.; GINTHER O.J. Functional interrelationships between follicles greater than $4 \mathrm{~mm}$ and the follicle-stimulating hormone surge in heifers. Biology of Reproduction, Madison, v.57, p.1066-1073, 1997.

GIBBONS J.R.; WILTBANK M.C.; GINTHER O.J. Relationship between follicular development and the decline in the follicle-stimulating hormone surge in heifers. Biology of Reproduction, Madison, v.60, p.72-77, 1999.

GINTHER O.J.; KNOPF L.; KASTELIC J.P. Temporal associations among ovarian events in cattle during oestrous cycles with two and three follicular waves. Journal of Reproduction and Fertility, Cambridge, v.87, p.223-30, 1989.

GINTHER O. J.; WILTBANK M.C.; FRICKE P. M.; GIBBONS J.R.; KOT K. Selection of the dominant follicle in cattle. Biology of Reproduction, Madison, v.55, p.1187-1194, 1996.

GINTHER O.J.; KOT K.; KULICK L.J.; WILTBANK M.C. Emergence and desviation of follicle during the development of follicular waves in cattle. Theriogenology, New York, v.48, p.75-87, 1997.

GINTHER O.J.; BERGFELD D.R.; KULICK L.J.; KOT K. Selection of the dominant follicle in cattle: establishment of follicle deviation in less than 8 hours through depression of FSH concentration. Theriogenology, New York, v.52, p.1079-1093, 1999.

GINTHER O.J.; BERGFELD D.R.; KULICK L.J.; KOT K. Selection of the dominant follicle in cattle: role of twoway functional coupling between follicle-stimulating hormone and the follicle. Biology of Reproduction, Madison, v.62, p.920-927, 2000a. 
GINTHER O.J.; BERGFELD D.R.; KULICK L.J.; KOT K. Selection of the dominant follicle in cattle: role of estradiol. Biology of Reproduction, Madison, v.63, p.383-389, 2000b.

GINTHER O.J.; BERGFELT D.R.; BEG M.A.; KOT K. Follicle selection in cattle:relationships among growth rate, diameter ranking and capacity for dominance. Biology of Reproduction, Madison, v.65, p.345-350, 2001.

GOLDENBERG R.L.; BRIDSON W.E.; KOHLER P.O. Estrogen stimulation of progesterone synthesis by porcine granulosa cells in culture. Biochemical and biophysical research communications, Madison, v.11, p.101-107, 1972.

GONG J.C.; BRAMLEY T.A.; GUTIERREZ C.G.; PETERS A.R.; WEBB R. Effects of chronic treatment with a gonadotrophin-releasing hormone agonist on peripheral concentrations of $\mathrm{FSH}$ and $\mathrm{LH}$, and ovarian function In heifers. Journal of Reproduction and Fertility, Cambridge, v.105, p.263-270, 1995.

GONG J.C.; CAMPBELL B.K.; BRAMLEY T.A.; GUTIERREZ C.G; PETERS A.R.; WEBB R. Suppression in the secretion of $\mathrm{FSH}$ and $\mathrm{LH}$, and ovarian follicle development in heifers continuously infused with $\mathrm{GnRH}$ agonist. Biology of Reproduction, Madison, v.55, p.68-74, 1996.

GOODHAND K.L.; WATT R.G.; STAINES M.E.; HUTCHINSON J.S.M. BROADBENT P.J. In vivo oocyte recovery and in vitro embryo production from bovine donors aspirated at different frequencies or following FSH treatment. Theriogenology, New York, v.51, p.951-961, 1999.

HAGEMANN L.J. Influence of the dominant follicle on oocytes from subordinate follicles. Theriogenology, New York, v.51, p.449-459, 1999.

HASLER J.F.; HENDERSON W.B.; HURTGEN P.J.; JIN Z.Q.; MCCAULEY A.D.; MOWER S.A.; NEELY B.; SHUEY L.S.; STOKES J.E.; TRIMMER S.A. Production, freezing and transfer of bovine IVF embryos and subsequent calving results. Theriogenology, New York, v.43, p.141152, 1995.

HENDRIKSEN P.J.M.; VOS P.L.A.M.; STEENWENG W.N.M.; BEVERS M.M.; DIELEMAN S.J. Bovine follicular development and its effect on the in vitro competence of oocytes. Theriogenology, New York, v.53, p.11-20, 2000.

HILLIER S.G. Paracrine control of follicular estrogen synthesis. Seminars in Reproductive Endocrinology, New York, v.9, p.332-340, 1991.

KO J.C.H.; KASTELIC J.P.; DEL CAMPO M.R.; GINTHER O.J. Effects of a dominant follicle on ovarian follicular dynamics during the oestrus cycle in heifers. Journal of Reproduction and Fertility, Cambridge, v.91, p.511-519, 1991.

KRUIP T.A.M.; BONI R.; WURTH Y.A.; ROELOFSEN M.W.M.; PIETERSE M.C. Potencial use of ovum pick-up for embryo production and breeding cattle. Theriogenology, New York, v.42, p.675-684, 1994.
KULICK L.J.; KOT K.; WILTBANK M.C.; GINTHER O.J. Follicular and hormonal dynamics during the first follicular wave in heifers. Theriogenology, New York, v.52, p.913-921, 1999.

LONERGAN P.; MONAGHAN P.; RIZOS D.; BOLAND M.P.; GORDON I. Effect of follicle size on bovine oocyte quality and developmental competence following maturation, fertilization, and culture in vitro. Molecular Reproduction and Development, New York, v.37, p.48-53, 1994.

LOONEY C. R.; LINDSEY B.R.; GONSETH C.L.; JOHNSON D.L. Commercial aspects of oocyte retrieval and in vitro fertilization (IVF) for embryo production in problem cows. Theriogenology, New York, v.41, p.67-72, 1994.

LUCY M.C.; SAVIO J.D.; BADINGA L.; DE LA SOTA R.L.; THATCHER W.W. Factors that affect ovarian follicular dynamics in cattle. Journal of Animal Science, Savoy, v.70, p.3615-26, 1992.

MACHATKOVÁ M.; JOKESOVÁ E.; PETELÍKOVÁ J.; DVORÁCEK V. Developmental competence of bovine embryo derived from oocytes collected at various stages of the estrous cycle. Theriogenology, New York, v.45, p.801-810 1996.

MACHATKOVÁ M, KRAUSOVÁ K, JOKESOVÁ E, TOMANEK M. Developmental competence of bovine oocytes: effects of follicle size and the phase of follicular wave on in vitro embryo production. Theriogenology, New York, v.15, p.329-335, 2004.

MEINTJES M.; BELLOW M.S.; BROUSSARD J.R.; PAUL J.B.; GODKE R.A. Transvaginal aspiration of oocytes from hormone-treated pregnant beef for in vitro fertilization. Journal of Animal Science, Savoy, v.73, p.967-974, 1995.

MIHM M.; AUSTIN E.J.; GOOD T.E.M.; IRELAND J.L.H.; KNIGHT P.G.; ROCHE J.F.; IRELAND J.J. Identification of potential intrafollicular factors involved in selection of dominant follicles in heifers. Biology of Reproduction, Madison, v.63, p.811-819, 2000.

MURDOCH W. J. Inhibition by oestradiol of oxidative stress-induced apoptosis in pig ovarian tissues. Journal of Reproduction and Fertility, Cambridge, v.114, p.127130, 1998.

PAVLOK A.; LUCANS-HAHN, NIEMANN H. Fertilization and developmental competence of bovine oocytes derived from different categories of antral follicles. Molecular Reproduction and Development, New York, v.31, p.63-67, 1992.

PIERSON R.A.; GINTHER O.J. Ultrassonic imaging of the ovaries and uterus in cattle. Theriogenology, New York, v.29, p.21-37, 1988.

PIETERSE M.C.; VOS P.L.A.M.; KRUIP T.A.M.; TAVERNE M.A.M. Tranvaginal ultrasound guided follicular aspiration of bovine ovaries. Theriogenology, New York, v.35, p.19-24, 1991.

RODRIGUES C.F.M.; GARCIA J.M. Fecundação in vitro em bovinos: aplicação comercial. Arquivos da Faculdade de Veterinária da UFRGS, Porto Alegre, v.28, p.186-187, 2000. 
SARTORI R.; FRICKE P.M.; FEREIRA J.C.; GINTHER O.J.; WILTBANK M.C. Follicular deviation and acquisition of ovulatory capacity in bovine follicles. Biology of Reproduction, Madison, v.65, p.1403-1409, 2001.

SAVIO J.D.; KEENAN L.; BOLAND M.P.; ROCHE J.F. Pattern of growth of dominant follicles during the oestrous cycle of heifers. Journal of Reproduction and Fertility, Cambridge, v.83, p.663-671, 1988.

SENEDA M.M.; ESPER C.R.; GARCIA J.M.; de OLIVEIRA J.A.; VANTINI R. Relationship between follicle size and ultrasound-guided transvaginal oocyte recovery. Animal Reproduction Science, Amsterdam, v.67, p.37-43, 2001.

SIROIS J.; FORTUNE J.E. (1990). Lengthening of the bovine estrous cycle with low levels of exogenous progesterone: a model for studying ovarian folicular dominance. Endocrinology, Bethesda, v.127, p.916-925.

STOCK A.E.; SMITH L. (1996). Developmental of bovine oocytes from small follicles is enhanced after maturation in medium conditioned by oocytes from big follicles. Biology of Reproduction, Madison, v.55, p.174. Abstr.

STUBBINGS R. B.; WALTON J. S. (1995). Effect of ultrasonically-guided follicle aspiration on estrous cycle and follicular dynamics in Holandesa cows. Theriogenology, New York, v.43, p.705-712.
SUNDERLAND S.J.; CROWE M.A.; BOLAND M.P.; ROCHE J.F.; IRELAND J.J. Selection, dominance and atresia of follicles during the oestrous cycle of heifers. Journal of Reproduction and Fertility, Cambridge, v.101, p.547-555, 1994.

WATANABE M.R. Aspiração in vivo de oócitos em fêmeas Nelore de diferentes idades reprodutivas mediante punção vaginal guiada por ultra-som. Jaboticabal, 2002. 48p. DISSERTAÇÃO (Mestrado) FCAV - UNESP - Jaboticabal.

WIT A.A.C.; WURTH Y.A.; KRUIP T.A.M. Effect of ovarian and follicle quality on morphology and developmental capacity of the bovine cumulus-oocyte complex. Journal of Animal Science, Savoy, v.8, p.1277-1283, 2000.

XU Z.Z.; GARVERICK H.A.; SMITH G.H.; SMITH M.F.; HAMILTON S.A.; YOUNGQUIST R.S. Expression of FSH and $\mathrm{LH}$ receptor messenger ribonucleic-acids in bovine follicles during the first follicular wave. Biology of Reproduction, Madison, v. 53, p.951-957, 1995.

YUAN W.; BAO B.; GARVERICK H.A.; YOUNQUIST R.S.; LUCY, M.C. Follicular dominance in cattle is associated with devergent patterns of ovarian gene expression for insulin-like growth factor (IGF-I), IGF-II and IGF binding protein-2 in dominant and subordinate follicles. Domestic animal endocrinology, New York, v.15, p.55-63, 1998.

Recebido para publicação: $\quad$ 03/11/2005 Aprovado: $15 / 01 / 2006$ 